\title{
FÓSFORO ORGÂNICO EM SOLOS SOB AGROSSISTEMAS DE $\operatorname{CACAU}^{(1)}$
}

\author{
Francisco Costa Zaia ${ }^{(2)}$, Antonio Carlos da Gama-Rodrigues ${ }^{(3)}$, Emanuela \\ Forestieri da Gama-Rodrigues ${ }^{(3)}$ \& Regina Celes Rebouça Machado ${ }^{(4)}$
}

\section{RESUMO}

\begin{abstract}
A compreensão de parte do ciclo do $P$ orgânico (Po) no solo poderá fornecer subsídios para o manejo eficiente da fertilização fosfatada no sistema de agricultura de baixos insumos na região tropical. O objetivo deste trabalho foi avaliar a variação de frações de Po em duas classes de solos sob diferentes agrossistemas de cacau no sul da Bahia. O Po total variou de 7,8 a 36,3 \% do P total extraído, com teor médio de $193,3 \mathrm{mg} \mathrm{kg}^{-1}$. O teor médio de Po lábil foi de $15,2 \mathrm{mg} \mathrm{kg}^{-1}$, com variação de $33,1 \mathrm{a}$ 81,9 \% do $P$ lábil total. Nos agrossistemas de cacau, o grupo de Latossolos apresentou menor teor de Po total, Po lábil e P microbiano do que o grupo de Cambissolos. No grupo de Latossolos, os solos sob agrossistemas de cacau apresentaram maior teor de Po total, $\mathbf{P}$ microbiano e $\mathbf{P}$ disponível, mas menor teor de Po lábil do que o solo sob floresta natural. O P disponível correlacionou-se positivamente com o Po (total, lábil e microbiano), e o Po total com o P microbiano. O Po lábil predominou amplamente sobre a fração inorgânica lábil, especialmente no grupo de Latossolos.
\end{abstract}

Termos de indexação: Mata Atlântica, disponibilidade de fósforo, frações lábeis de fósforo, biomassa microbiana.

\footnotetext{
(1) Parte da Dissertação do primeiro autor, apresentado ao Curso de Pós-Graduação em Produção Vegetal - CCTA/UENF. Recebido para publicação em outubro de 2007 e aprovado em setembro de 2008.

${ }^{(2)}$ Doutorando do Curso de Produção Vegetal da Universidade Estadual do Norte Fluminense Darcy Ribeiro - UENF. CEP 28013602 Campos dos Goytacazes (RJ). E-mail zaia@uenf.br

(3) Professor do Laboratório de Solos do Centro de Ciências e Tecnologias Agropecuárias - CCTA, UENF. E-mails: tonygama@uenf.br; emanuela@uenf.br

${ }^{(4)}$ Pesquisadora do Almirante Centro de Estudos do Cacau. CEP 45630-000 Itajuipe (BA). E-mail regina.machado@effen.com
} 


\title{
SUMMARY: ORGANIC PHOSPHORUS IN SOILS UNDER COCOA AGROSYSTEMS
}

\begin{abstract}
Understanding the soil organic $P(P o)$ cycle is important to improve the $P$ fertilization management in low-input tropical agricultural systems. The aim of this study was to evaluate Po content and labile $P$ fractions, and microbial biomass $P$ in soils under different cocoa agroecosystems. Mean total Po was $193 \mathrm{mg} \mathrm{kg}^{-1}$ and accounted for 7.8 to $36.3 \%$ of the total extracted P. Mean labile Po was $15 \mathrm{mg} \mathrm{kg}^{-1}$ and accounted for 33.1 to $81.9 \%$ of the total labile P. In cocoa agrosystems, the total Po, labile Po and microbial P contents were lower in the Oxisol than in the Inceptsol group. In the Oxisol group, in soils under cocoa agrosystems, the total Po, microbial $P$ and available $P$ contents were higher and the labile $P o$ lower than in the soil under natural forest. Available $P$ was positively correlated with $P o$ (total, labile and microbial), and total Po was positively related to microbial $P$. The labile Po fraction was far higher in the labile inorganic fraction, especially in the Oxisol group.
\end{abstract}

Index term: Atlantic Forest, available P, labile P fraction, microbial biomass.

\section{INTRODUÇÃO}

A importância relativa do $\mathrm{P}$ orgânico $(\mathrm{Po})$ na nutrição das plantas aumenta quando há deficiência de $\mathrm{P}$, resultante dos baixos teores totais e, ou, forte adsorção de $\mathrm{P}$ pelos oxiidróxidos de $\mathrm{Fe}$ e $\mathrm{Al}$ no solo. Nessas condições, a ciclagem de formas orgânicas mais lábeis é acelerada, sendo mais importante em solos tropicais altamente intemperizados (Silva \& Mendonça, 2007). Nesses solos, o conteúdo de Po seria maior naqueles mais argilosos (Cade-Menun, 2005), com maior teor de C orgânico (Gressel \& McColl, 1997; Cunha et al., 2007) e menor pH (Cunha et al., 2007).

Nos sistemas agroflorestais e florestais, por promoverem uma grande acumulação de matéria orgânico no solo, o P disponível está estreitamente associado ao Po lábil (Szott \& Melendez, 2001; Lehmann et al., 2001; George et al., 2002; Comerford et al., 2006). Nesses sistemas, além das formas lábeis de Po, o conteúdo de $\mathrm{P}$ da biomassa microbiana do solo possui também uma estreita relação com o $\mathrm{P}$ disponível (Grieson et al., 2004; Zaia, 2005), por constituir a fração ativa e de rápido turnover do ciclo de $\mathrm{P}$ no solo. Assim, ambas as frações de Po lábil, e de $\mathrm{P}$ microbiano constituiriam importantes reservatórios de $\mathrm{P}$, diminuindo a capacidade de adsorção da fase mineral do solo e, conseqüentemente, o aumento da disponibilidade de $\mathrm{P}$ para as plantas.

No Brasil, o cacau, por se caracterizar como uma cultura de subbosque, seja sob floresta natural, seja sob floresta homogênea, constitui agrossistema adequado para estudos de ciclagem de nutrientes (Gama-Rodrigues, 2004). A combinação do cacaueiro com espécies não lenhosas (banana, mandioca, etc.) e espécies lenhosas (eritrina, gliricídia, etc.) seria um bom exemplo da compatibilidade e complementaridade de diferentes espécies e ao mesmo tempo de sustentabilidade de sistemas de produção multiestratificados (Muller \& Gama-Rodrigues, 2007). Nesse sentido, alguns trabalhos de ciclagem de nutrientes têm sido realizados procurando dar ênfase ao ciclo da matéria orgânica (Fassbender et al., 1988; Santana et al., 1990; Fontes, 2006) ou ao ciclo hidrológico (Gama-Rodrigues \& Miranda, 1991a,b) e à sua contribuição no fornecimento de nutrientes. Contudo, tem sido pouco estudado o papel do Po na disponibilidade de $\mathrm{P}$ para a cultura do cacaueiro (Acquaye, 1963), apesar de esse agrossistema promover acumulação de matéria orgânica no solo (Fontes, 2006) e do $\mathrm{P}$ ser considerado o principal fator nutricional limitante à expansão dessa cultura em solos mais intemperizados (Santana et al., 1988). Assim, estudos sobre Po poderiam fornecer importantes subsídios para o desenvolvimento de técnicas de manejo da fertilização fosfatada, que sejam ecológica e economicamente sustentáveis.

O objetivo deste trabalho foi avaliar a variação de frações de Po em duas classes de solos sob diferentes agrossistemas de cacau no sul da Bahia.

\section{MATERIAL E MÉTODOS}

O trabalho foi realizado na região cacaueira do município de Itajuípe, no sul da Bahia. A vegetação foi classificada como Floresta Ombrófila Densa das Terras Baixas (Veloso et al., 1991). O clima da região, pela classificação de Köppen, é do tipo Af, caracterizado por uma pluviosidade média anual de $1.500 \mathrm{~mm}$, sem estação seca definida, e a temperatura média anual é de $26^{\circ} \mathrm{C}$ (Estação Climatológica Almirante Centro de Estudos de Cacau, dados não publicados).

Estudaram-se cinco agrossistemas de cacau: 1 . cacau renovado eritrina (Erythrina glauca); 2 - cacau renovado cabruca; 3 - cacau antigo cabruca; 4 - cacau antigo eritrina; e 5 - cacau em jardim clonal adensado. A idade dos agrossistemas 1, 2 e 4 era de 30 anos, mas nos dois primeiros houve renovação de copa por enxertia com idade de três anos, sem recepa da copa anterior. Nos agrossistemas 3 e 5 , a idade era de 70 e 
15 anos, respectivamente. O nível de sombreamento foi maior que $30 \%$, à exceção do agrossistema 5 . Nos agrossistemas de 1 a 4 o espaçamento do cacaueiro foi de 3 x $2 \mathrm{~m}$. Para as árvores de sombra, o espaçamento da eritrina foi de 24 × 24 m distribuídas em quincôncio, enquanto na cabruca as árvores estavam espaçadas aleatoriamente. No agrossistema 5, o espaçamento do cacaueiro foi em linha dupla de $3 \times 1,5 \times 1,3 \mathrm{~m}$, sob sombra de eritrina e gliricídia (Gliricidia sepium) em espaçamento de 30 x 30 m, em quincôncio. Em todos os agrossistemas não tem havido fertilização mineral ou orgânica ( $30 \mathrm{~kg} \mathrm{ha}^{-1}$ de $\left.\mathrm{P}_{2} \mathrm{O}_{5}\right)$ há mais de 10 anos. Uma Floresta Ombrófila Densa Secundária Tardia serviu de referencial aos outros sistemas.

Neste trabalho, a cabruca foi o sistema de cultivo do cacau como sub-bosque de floresta natural. Baseouse na substituição da vegetação rasteira e das árvores de menor porte, que ofereciam maior competitividade ao cacaueiro, permanecendo somente aquelas que poderiam ser utilizadas como sombra provisória e, em alguma situação, até como sombra definitiva. Após esta operação, selecionaram-se árvores de copa alta e pouco densa para o sombreamento definitivo e, então, derrubaram-se as restantes.

$\mathrm{Na}$ floresta natural e nos agrossistemas de 1 a 3 , os solos foram classificados como Latossolos VermelhoAmarelo distróficos e, nos agrossistemas 4 e 5, como Cambissolos Háplicos Tb eutróficos, todos em relevo ondulado. Para a caracterização da fertilidade do solo e dos teores de Po lábil e Po total e, de C e P da biomassa microbiana, em cada cobertura vegetal, foi delimitada uma parcela fixa de $1.500 \mathrm{~m}^{2}$. Em cada parcela foram coletadas quatro amostras compostas, sendo cada uma constituída de quinze amostras simples ao acaso nas entrelinhas de plantio, na camada de $0-5 \mathrm{~cm}$, em maio de 2004. A composição granulométrica e os atributos químicos foram determinados de acordo com Embrapa (1997), exceção do conteúdo de P total, estimado a partir de digestão nítrico-perclórica (Bataglia et al., 1983) e de $\mathrm{C}$ orgânico dosado por oxidação com $\mathrm{K}_{2} \mathrm{Cr}_{2} \mathrm{O}_{7}$ 1,25 $\mathrm{mol} \mathrm{L}^{-1} \mathrm{em}$ meio ácido (Anderson \& Ingram, 1996) (Quadro 1). Foram também coletadas amostras de solo na camada de $0-10 \mathrm{~cm}$ para determinação dos teores das frações de Po e Pi.

A quantificação do Po total foi obtida empregandose o método de extração seqüencial (Bowman, 1989), enquanto o Po lábil pelo método de extração com $\mathrm{NaHCO}_{3}$ 0,5 mol L-1 (Bowman \& Cole, 1978). O Pi foi determinado após clarificação dos extratos com carvão ativo (Guerra et al., 1996). O teor de Pi nos extratos ácidos e alcalinos foi determinado pelo método de Murphey \& Riley (1962). O método da fumigaçãoextração foi utilizado para estimar o C (Vance et al., 1987) e o P (Brookes et al., 1984) da biomassa microbiana do solo.

Em cada cobertura vegetal, na comparação entre as camadas de amostragem para os teores das frações de Pi, Po e Pi + Po do solo, os dados foram submetidos à análise de variância como em delineamento inteiramente casualizado com quatro repetições. Adotou-se o teste $\mathrm{F}$ a $5 \%$. Foram estabelecidas correlações de Pearson a 5 \% entre as diferentes frações de $\mathrm{P}$ e alguns atributos químicos do solo.

Cada cobertura vegetal, foi considerada um tratamento de efeito fixo. Assim, os dados das frações de Po (total, lábil e microbiano) e Pi, de P disponível (Mehlich-1), de C orgânico e da biomassa microbiana e mais argila também foram submetidos à análise de componentes principais (ACP) com o objetivo de sintetizar sua variação multidimensional em um diagrama, ordenando-os nos componentes, de acordo com suas similaridades em torno das variáveis utilizadas (Ter Braak, 1986). As variáveis foram padronizadas para reduzir efeitos de escala. A ACP transforma um conjunto original de variáveis em outro conjunto de dimensão equivalente. Assim, as amostras (coberturas vegetais) e as variáveis (diferentes frações de P, C e argila) foram transformadas em coordenadas, que correspondem à sua projeção nos eixos de ordenação, ou autovetores, representando o peso de cada variável sobre cada componente (eixo) e funciona como coeficiente de correlação, que varia de -1 até +1 . As variáveis com elevado autovetor no primeiro eixo tendem a ter autovetor inferior no segundo eixo. Neste trabalho, considerou-se o autovetor $<0,7$ como de baixa associação para a interpretação dos componentes principais (Wick et al., 1998). Na maioria dos estudos, como no caso deste trabalho, apenas os dois primeiros componentes são utilizados, sendo considerados suficientes para explicar os dados e facilitar a interpretação do gráfico em duas dimensões (Gomes et al., 2004).

Um ponto qualquer plotado no diagrama (representando uma dada cobertura vegetal) pode ser relacionado com cada seta (teores de $\mathrm{P}, \mathrm{C}$ ou argila), por meio de uma perpendicular partindo da linha da seta até o referido ponto. A ordem nas quais os pontos se projetam na seta, da sua extremidade até sua origem, dá uma indicação dessa relação. Coberturas vegetais com sua projeção perpendicular próxima, ou além da ponta da seta, são mais positivamente correlacionadas e influenciadas pela variável em questão. Aqueles na extremidade oposta são influenciados em menor grau. O ângulo de inclinação de cada seta com relação a cada eixo indica quão estreitamente correlacionado está o atributo com este eixo (Alvarenga \& Davide, 1999).

\section{RESULTADOS E DISCUSSÃO}

\section{Cobertura vegetal}

A textura e o nível de fertilidade do solo variaram amplamente entre as coberturas vegetais (Quadro 1). $\mathrm{O}$ grupo de Cambissolos apresentou menor acidez e maior nível de fertilidade do solo do que o grupo de Latossolos. Nesse grupo de solos, maior acidez e o 
menor nível de fertilidade ocorreram no solo sob floresta natural em relação aos agrossistemas de cacau. Entretanto, apenas para C orgânico praticamente não houve variação entre a floresta natural e os agrossistemas de cacau. Os maiores teores de argilas ocorreram no grupo de Latossolos, sendo o solo mais argiloso sob floresta natural. Com base na classificação proposta por Alvarez V. et al. (1999), considerando os teores de argila, os teores de P disponível (Mehlich-1) foram baixos no grupo de Latossolos, enquanto no grupo de Cambissolos variaram de médio a muito alto.

A relação $(\mathrm{Pi}+\mathrm{Po}) / \mathrm{P}$ total indica a taxa de recuperação do método em relação ao $\mathrm{P}$ total do solo por digestão. A taxa de recuperação variou de 40 a $169 \%$ (Quadro 2). As taxas de recuperação encontradas por Condron et al. (1990) variaram entre 30 e $107 \%$ do P total, por Guerra et al. (1996) entre 48 e $109 \%$, e por Cunha et al (2007) entre 50 e $82 \%$.
Os maiores teores das frações de P (inorgânico e orgânico) foram encontrados no grupo de Cambissolos, sob cacau antigo e eritrina e sob cacau em jardim clonal adensado (Quadro 2). O Pi predominou na composição do $\mathrm{P}$ total $(\mathrm{Pi}+\mathrm{Po})$ nos solos sob todas as coberturas vegetais. O Po total representou de 7,8 a $36,3 \%$ do $\mathrm{P}$ total extraído. O Latossolo sob cacau renovado e cabruca apresentou a menor relação $\mathrm{Po} / \mathrm{P}$ total, enquanto a maior participação do Po na composição do $\mathrm{P}$ total foi no Latossolo sob cacau antigo e cabruca. Cunha et al. (2007) e Zaia (2005), usando o mesmo método de determinação de Po deste trabalho, encontraram para Latossolos e Cambissolos variação de Po total de 22,6 a 39,6 \% em solos florestais e de 14,6 a $24,1 \%$ em solos de pastagens.

O teor médio de Po total foi de $193,3 \mathrm{mg} \mathrm{kg}^{-1}$. No grupo de Latossolos, o teor médio foi de $76,7 \mathrm{mg} \mathrm{kg}^{-1}$, enquanto no grupo de Cambissolos foi de $426,4 \mathrm{mg} \mathrm{kg}^{-1}$. Esses teores de Po total estão dentro da faixa de valores

Quadro 1. Características físicas e químicas do solo, na camada de 0-5 cm, sob agrossistemas de cacau

\begin{tabular}{|c|c|c|c|c|c|c|c|c|c|c|}
\hline Solo & Cobertura & Areia & Silte & Argila & $\mathrm{pH} \mathrm{H} \mathrm{H}_{2} \mathrm{O}$ & $\mathbf{P t}^{(2)}$ & $\mathbf{P}^{(3)}$ & Al & SB & C \\
\hline & & & \multicolumn{2}{|l|}{$\mathrm{g} \mathrm{kg}^{-1}$} & & \multicolumn{2}{|c|}{$-\mathrm{mg} \mathrm{kg}^{-1}$ - } & \multicolumn{2}{|c|}{$-\mathrm{mmol}_{\mathrm{c}} \mathrm{kg}^{-1}-$} & $\mathrm{g} \mathrm{kg}^{-1}$ \\
\hline Latossolo & Floresta natural & 300 & 110 & 590 & 3,8 & 585 & 4,3 & 19,2 & 27,0 & 41,15 \\
\hline Latossolo & Cacau ren. e eritrina ${ }^{(1)}$ & 410 & 280 & 310 & 4,6 & 690 & 7,7 & 2,7 & 63,7 & 39,72 \\
\hline Latossolo & Cacau ren. e cabruca & 450 & 120 & 430 & 4,9 & 715 & 6,7 & 0,8 & 83,8 & 40,19 \\
\hline Latossolo & Cacau antigo e cabruca & 440 & 230 & 330 & 5,4 & 736 & 6,8 & 0,2 & 91,0 & 39,52 \\
\hline Cambissolo & Cacau antigo e eritrina & 410 & 420 & 170 & 5,9 & 989 & 17,0 & 0,0 & 151,0 & 16,95 \\
\hline Cambissolo & Cacau jardim clonal & 540 & 340 & 120 & 6,0 & 1.289 & 73,3 & 0,0 & 192,0 & 29,21 \\
\hline
\end{tabular}

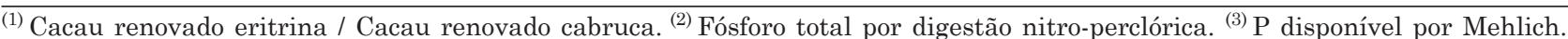

Quadro 2. Teores das frações de P inorgânico (Pi), orgânico (Po) e inorgânico + orgânico nas formas lábil e total do solo, na camada de $0-5 \mathrm{~cm}$, sob agrossistemas de cacau

\begin{tabular}{|c|c|c|c|c|c|c|c|}
\hline \multirow{2}{*}{ Solo } & \multirow{2}{*}{ Cobertura } & \multicolumn{2}{|c|}{$\mathbf{P i}$} & \multicolumn{2}{|c|}{ Po } & \multicolumn{2}{|l|}{$\mathbf{P i}+\mathbf{P o}$} \\
\hline & & Total & Lábil & Total & Lábil & Total & Lábil \\
\hline Latossolo & Floresta natural & $212,8(82,6)^{(1)}$ & $3,9(18,7)^{(2)}$ & $44,8(17,4)^{(1)}$ & $17,1(81,3)^{(2)}$ & $257,6 \quad(44)^{(3)}$ & 21,0 \\
\hline Latossolo & Cacau renovado e eritrina & $338,2 \quad(84,8)$ & $6,1 \quad(28,9)$ & $60,4 \quad(15,2)$ & $15,1 \quad(71,1)$ & $398,6(58)$ & 21,2 \\
\hline Latossolo & Cacau renovado e cabruca & $262,0 \quad(92,2)$ & $4,2 \quad(24,7)$ & $22,3 \quad(7,8)$ & $12,8(75,3)$ & $284,3(40)$ & 17,0 \\
\hline Latossolo & Cacau antigo e cabruca & $314,2 \quad(63,7)$ & $5,8(34,7)$ & $179,3 \quad(36,3)$ & $10,8(65,3)$ & $493,5 \quad(67)$ & 16,6 \\
\hline Cambissolo & Cacau antigo e eritrina & $1207,0 \quad(72,3)$ & $15,0 \quad(47,9)$ & $461,8 \quad(27,4)$ & $16,3(52,1)$ & $1.668,8(169)$ & 31,3 \\
\hline Cambissolo & Cacau jardim clonal & $881,9 \quad(69,3)$ & $38,6 \quad(66,9)$ & $391,0 \quad(30,7)$ & $19,1 \quad(33,1)$ & $1.272,9(99)$ & 57,7 \\
\hline Média & & $536,0 \quad(73,5)$ & $12,2 \quad(44,5)$ & $193,3(26,5)$ & $15,2(55,5)$ & $729,3(87)$ & 27,4 \\
\hline CV (\%) & & 69,7 & 101,3 & 89,7 & 18,0 & 74,3 & 52,4 \\
\hline
\end{tabular}

(1) Percentual em relação a Pi + Po total. ${ }^{(2)}$ Percentual em relação a Pi + Po lábil. ${ }^{(3)}$ Recuperação percentual em relação ao P total estimado a partir de digestão nítrico-perclórica. 
encontrados para Cambissolos, que variaram entre 87 e 771,5 mg kg-1 (Duda, 2000; Cunha et al., 2007), e para Latossolos, que variaram entre 22 e $119,9 \mathrm{mg} \mathrm{kg}^{-1}$ (Guerra et al., 1996; Zaia, 2005; Cunha et al., 2007).

O teor médio de Po lábil foi de $15,2 \mathrm{mg} \mathrm{kg}^{-1}$. Nos grupos de Latossolos e de Cambissolos, os teores médios desta fração foram de 14,0 e $17,7 \mathrm{mg} \mathrm{kg}^{-1}$, respectivamente. Verifica-se, assim, que a maior diferença entre as duas classes de solos foi para o Po total. Entretanto, esta tendência não foi verificada para Pi (total e lábil), e, no grupo de Latossolos, as frações de Pi corresponderam em torno de $20 \%$ dos teores dessas frações em relação ao grupo de Cambissolos (Quadro 2).

O balanço entre Pi lábil e Po lábil em relação ao P lábil total variou entre os tipos de solos (Quadro 2). A fração Po lábil foi maior do que o Pi lábil, ao contrário do balanço de Pi e Po em relação ao P total. No grupo de Latossolos, sob floresta natural 81,3 \% do P lábil e de 65,3 a $75,3 \%$ sob agrossistemas de cacau foram constituídos por Po lábil; entretanto, no grupo de Cambissolos a proporção do Po lábil variou de 33,1 a $52,1 \%$. Na região norte-fluminense, a proporção de Po lábil em Cambissolos sob florestas naturais foi de 86,3 e de $68,8 \%$ em Latossolos sob eucalipto, leguminosas florestais e floresta natural (Zaia, 2005; Cunha et al., 2007).

O predomínio de Po lábil em relação ao Pi lábil no grupo de Latossolos (Quadro 2), encontrado também por Guerra et al. (1996), indicou que a disponibilidade de $\mathrm{P}$, em curto prazo, não é completamente acessada por determinações de $\mathrm{P}$ disponível (Novais et al., 2007). Em solos de avançado estádio de intemperismo, o $\mathrm{P}$ disponível está estreitamente relacionado às frações do Po (George et al., 2002; Grierson et al., 2004).

Para o P da biomassa microbiana, a amplitude de variação foi de $3,93 \mathrm{mg} \mathrm{kg}^{-1} \mathrm{de} \mathrm{P}$, no Latossolo sob floresta natural, a $17,88 \mathrm{mg} \mathrm{kg}^{-1}$ de $\mathrm{P}$, no Cambissolo sob cacau em jardim clonal adensado (Quadro 3). O grupo de Cambissolos apresentou valores médios de $\mathrm{P}$ microbiano 2,3 vezes superiores aos do grupo de Latossolos. O C da biomassa microbiana também apresentou ampla variação entre os solos; entretanto os maiores valores médios foram encontrados no grupo de Latossolos (471,7 $\mathrm{mg} \mathrm{kg}^{-1} \mathrm{de} \mathrm{C}$ ) do que no grupo de Cambissolos (305,5 $\left.\mathrm{mg} \mathrm{kg}^{-1} \mathrm{de} \mathrm{C}\right)$.

A maior eficiência da biomassa microbiana do solo em imobilizar $\mathrm{C}$ (Cbm/C orgânico) e $\mathrm{P}(\mathrm{Pbm} / \mathrm{Po}$ total), em média, foi encontrada no grupo de Latossolos (Quadro 3). A maior proporção de Po mineralizável, indicada pela relação Po lábil/Po total, também ocorreu no grupo de Latossolos sob cacau renovado e cabruca (57,5\%), floresta natural (38,2 \%), cacau renovado e eritrina $(24,9 \%)$ e cacau antigo e cabruca $(6,0 \%)$. Já no grupo de Cambissolos, a proporção de Po mineralizável foi de 3,5\% para cacau antigo e eritrina e de $4,9 \%$ para cacau em jardim clonal adensado. Zaia (2005) encontrou teores de P microbiano na faixa de 1,96 a 10,54 $\mathrm{mg} \mathrm{kg}^{-1}$ em Latossolos sob pastagem, leguminosa arbórea e floresta natural. Em Argissolos, os valores de $\mathrm{P}$ microbiano estão na faixa de 7,7 a $13,7 \mathrm{mg} \mathrm{kg}^{-1}$ sob pastagens (Guerra et al., 1995), de 7,6 a $10,8 \mathrm{mg} \mathrm{kg}^{-1}$ sob plantios de leguminosas herbáceas perenes (Duda et al., 2003) e de 1,0 a 7,5 mg kg-1 sob plantios de milho (Matos et al., 2006).

Os dois primeiros componentes principais $(\mathrm{CP})$ foram utilizados para explicar a variação total entre os solos, quando foram analisadas conjuntamente as frações de Pi (total e lábil), Po (total, lábil e microbiano) e P disponível (Mehlich-1), e mais os teores de argila, $\mathrm{C}$ orgânico e $\mathrm{C}$ da biomassa microbiana dos solos. $\mathrm{O}$ primeiro componente explicou $75,8 \%$ e o segundo $12,3 \%$, perfazendo $88,1 \%$ da variância total acumulada entre os solos. A dispersão gráfica dos solos sob as diferentes coberturas (Figura 1) demonstra dissimilaridade entre os dois grupos de solos analisados: o grupo de Latossolos localizados à esquerda do diagrama de ordenação, ao contrário do grupo de Cambissolos, que ficou localizado à direita.

Quadro 3. Teores das frações de P da biomassa microbiana (Pbm) e de C da biomassa microbiana (Cbm), relações de $\mathrm{C}$ microbiano para $\mathrm{C}$ orgânico $(\mathrm{Cbm} / \mathrm{C})$ e de $\mathrm{P}$ microbiano para Po total $(\mathrm{Pbm} / \mathrm{Pot})$ do solo, na camada de 0-5 cm, sob agrossistemas de cacau

\begin{tabular}{|c|c|c|c|c|c|}
\hline Solo & Cobertura & $\mathrm{Pbm}$ & Cbm & $\mathrm{Cbm} / \mathrm{C}$ & $\mathrm{Pbm} / \mathrm{Pot}$ \\
\hline & & \multicolumn{2}{|c|}{$\mathrm{mg} \mathrm{kg}^{-1}$} & 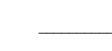 & - \\
\hline Latossolo & Floresta natural & 3,93 & 688,3 & 1,67 & 9 \\
\hline Latossolo & Cacau renovado e eritrina & 6,00 & 437,2 & 1,10 & 10 \\
\hline Latossolo & Cacau renovado e cabruca & 7,86 & 453,5 & 1,13 & 36 \\
\hline Latossolo & Cacau antigo e cabruca & 8,36 & 307,7 & 0,78 & 5 \\
\hline Cambissolo & Cacau antigo e eritrina & 12,39 & 395,3 & 2,33 & 3 \\
\hline Cambissolo & Cacau jardim clonal & 17,88 & 215,6 & 0,74 & 5 \\
\hline Média & & 9,40 & 416,3 & 1,29 & 11 \\
\hline $\mathrm{CV}(\%)$ & & 48,7 & 35,1 & 43,0 & 102,6 \\
\hline
\end{tabular}


No grupo de Latossolos, verifica-se que o solo sob floresta natural apresentou alto grau de dissimilaridade em relação aos agrossistemas de cacau, porém, dentre esses agrossistemas, houve maior similaridade apenas entre o cacau renovado e a eritrina e o cacau renovado e a cabruca. No grupo de Cambissolos, houve baixa similaridade entre os dois agrossistemas de cacau.

As variáveis mais associadas ao $\mathrm{CP} 1$ foram as frações de $\mathrm{P}$ microbiano, Po total, $\mathrm{Pi}$ lábil, $\mathrm{Pi}$ total e $\mathrm{P}$ disponível, nessa ordem, com autovetores positivos, e argila, C orgânico e C microbiano, com autovetores negativos (Quadro 4 e Figura 1). O CP2 serve para realçar as diferenças de Po lábil e, até certo ponto, de $\mathrm{C}$ microbiano, dentre os diferentes solos. Esses resultados revelam que, das diferentes frações de $\mathrm{P}$ analisadas, o Po lábil foi de pequeno significado $(<0,70)$ na distinção dos solos (Quadro 4). Observa-se uma grande sobreposição dos efeitos entre as frações de $\mathrm{P}$ associadas ao CP1. O P disponível correlacionou-se positivamente com Po total $(\mathrm{r}=0,634 ; \mathrm{p}<0,01)$, Po lábil $(r=0,652 ; p<0,01)$ e $P$ microbiano $(r=0,901$; $\mathrm{p}<0,01)$, e também houve correlação positiva entre Po total e $\mathrm{P}$ microbiano $(\mathrm{r}=0,837$; $\mathrm{p}<0,01)$. Entretanto, a argila correlacionou-se negativamente com Po total $(\mathrm{r}=-0,843 ; \mathrm{p}<0,01), \mathrm{P}$ microbiano $(\mathrm{r}=$ $0,872 ; p<0,01)$ e P disponível $(r=-0,689 ; p<0,01)$. O C orgânico também se correlacionou negativamente com Po total $\left(r=-0,926^{* *}\right)$ e $\mathrm{P}$ microbiano $(\mathrm{r}=-0,673$; $\mathrm{p}<0,01)$. Ao contrário das frações de $\mathrm{P}$, houve correlação positiva entre C orgânico e argila $(\mathrm{r}=0,738$; $\mathrm{p}<0,01)$.

Essas correlações sugerem que a acumulação do Po nos solos estaria mais estreitamente associada aos teores de $\mathrm{P}$ disponível que aos teores de $\mathrm{C}$ orgânico e de argila. Como esperado, a acumulação de C estaria estreitamente associada à argila. Isto explica, em parte, a associação negativa encontrada entre $\mathrm{C}$

Quadro 4. Cargas relativas das diferentes variáveis associadas aos componentes principais (CP) de solos, na camada de $0-5 \mathrm{~cm}$, sob agrossistemas de cacau (as cargas sublinhadas foram usadas para interpretar cada componente principal)

\begin{tabular}{lll}
\hline Variável & CP1 & CP2 \\
\hline Pi total & $\underline{0,895}$ & $-0,281$ \\
Pi lábil & $\underline{0,901}$ & $-0,004$ \\
Po total & $\underline{0,934}$ & $-0,110$ \\
Po lábil & 0,522 & $-\underline{0,686}$ \\
P microbiano & $\underline{0,951}$ & 0,140 \\
P disponível & $\underline{0,836}$ & 0,052 \\
C orgânico & $-\underline{0,813}$ & 0,347 \\
C microbiano & $-\underline{0,760}$ & $-0,637$ \\
Argila & $-0,932$ & $-0,203$ \\
\hline
\end{tabular}

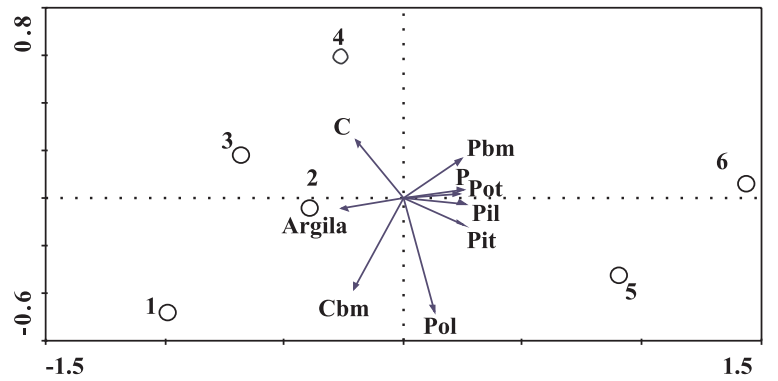

Figura 1. Diagrama de ordenação produzido pela análise de componentes principais dos atributos do solo. Pot = Po total; Pol = Po lábil; Pit = Pi total; Pil = Pi lábil; $\mathbf{P b m}=\mathbf{P}$ da biomassa microbiana; $\mathbf{P}=\mathbf{P}$ disponível; $\mathbf{C}=\mathbf{C}$ orgânico; $\mathbf{C b m}=\mathbf{C}$ da biomassa microbiana e argila. Grupo de Latossolos: coberturas 1 (floresta natural), 2 (cacau renovado e eritrina); 3 (cacau renovado e cabruca) e 4 (cacau antigo e cabruca). Grupo de Cambissolos: coberturas 5 (cacau antigo e eritrina) e 6 (cacau em jardim clonal adensado).

orgânico e as frações de Po (total e microbiano). Desse modo, constata-se um comportamento distinto de acumulação nos solos entre C orgânico e Po. Solos com elevados teores de $\mathrm{P}$ disponível tenderiam a ter proporcionalmente altos teores de Po. Como não houve fertilização fosfatada nos últimos dez anos, três fatores podem explicar concomitantemente a variação das diferentes formas de $\mathrm{P}$ nos solos estudados: o distinto grau de intemperismo entre os dois grupos de solos, no qual os Cambissolos são menos evoluídos, onde o ciclo geoquímico de $\mathrm{P}$ é ainda relevante, caracterizando esses solos como fonte de P. Nesse caso, como a quantidade de Pi poderia atender parcial ou totalmente a demanda de $P$ pelas plantas e pelos microrganismos, haveria baixas taxas de mineralização de $\mathrm{Po}_{0}$, proporcionando maior acumulação dessa fração de $\mathrm{P}$ no solo; a variação do teor de argila entre os solos, nos quais os mais argilosos apresentam baixos teores de $\mathrm{P}$ disponível devido ao elevado poder tampão e, ou, de adsorção de $\mathrm{P}$; e o ciclo biogeoquímico de $\mathrm{P}$, especialmente no grupo de Latossolos, onde os agrossistemas de cacau são mais eficientes que a floresta natural em manter maiores teores de Po e $\mathrm{P}$ disponível. Fontes (2006), estudando a ciclagem biogeoquímica nas mesmas coberturas vegetais deste trabalho, encontrou similaridade da biomassa de serapilheira acumulada entre a floresta natural e os agrossistemas de cacau. Contudo, o conteúdo de P na serapilheira dos agrossistemas de cacau foi maior do que o da floresta natural. Assim, a quantidade de $\mathrm{P}$ a ser incorporado ao solo, via processo de mineralização, é maior nos agrossistemas de cacau.

$\mathrm{Na}$ fase final da decomposição da serapilheira, o $\mathrm{P}$ do substrato, parte é mineralizado (Pi) e outra ainda está em forma orgânica, com elevado grau de recalcitrância (Gressel \& Mccoll, 1997). Esta fração 
de Po fará parte do processo de acumulação de Po estável do solo, enquanto o $\mathrm{P}$ mineralizado pode ser imobilizado na biomassa microbiana $(\mathrm{Pbm})$ e posteriormente fazer parte da fração de Po lábil, ou ser adsorvido na fase mineral do solo (Pi lábil) (Grierson et al., 2004). Na fase orgânica, parte do $P$ pode ser remineralizado, retornando à solução do solo. Na fase mineral, a maior parte do $\mathrm{P}$ tende a ser fixada, devido às baixíssimas taxas de dessorção. Entretanto, ânions orgânicos, derivados da decomposição da serapilheira, poderiam aumentar as taxas de dessorção de $\mathrm{P}$ como um resultado da troca de ligantes, elevando à disponibilidade de $\mathrm{P}$ (Wong et al., 2004). Assim, o P disponível seria dependente do balanço dos processos de mineralização/imobilização na fase orgânica, e de adsorção/dessorção na fase mineral, em relação à taxa de transferência de $\mathrm{P}$ da serapilheira para o solo.

\section{Camada de amostragem}

A variação entre os teores das frações de Pi (total e lábil), $\mathrm{Po}$ (total e lábil) e $\mathrm{P}$ total ( $\mathrm{Pi}+\mathrm{Po}$ ), na camada de $0-10 \mathrm{~cm}$, em cada cobertura vegetal, foi semelhante ao ocorrido na camada de $0-5 \mathrm{~cm}$. Contudo, os teores dessas frações de P na camada de $0-10 \mathrm{~cm}$ foram significativamente menores do que na camada de $0-5 \mathrm{~cm}$ em todas as coberturas vegetais (Quadro 5). Na camada de 0-10 cm, os teores médios de Po total e lábil foram de 134,9 e $8,3 \mathrm{mg} \mathrm{kg}{ }^{-1}$, respectivamente.
Esses teores corresponderam a uma redução de -30,2 e $-45,4 \%$, respectivamente, em relação a camada de 0-5 cm.

Estes resultados indicam que camadas de amostragem superiores a 0-5 cm proporcionam "efeito de diluição" na determinação das frações de P em solos de agrossistemas de cacau e de floresta natural. Nessas coberturas vegetais, a ciclagem biogeoquímica de $\mathrm{P}$ seria decorrente da deposição de resíduos da parte aérea na superfície do solo (Fontes, 2006), no qual a incorporação desses resíduos mediante o processo de decomposição ocorre predominantemente nos primeiros centímetros do perfil do solo. Além disso, na camada de $0-5 \mathrm{~cm}$ também ocorre uma grande quantidade de raízes finas e radicelas (Kummerow et al., 1982; Gama-Rodrigues \& Cadima-Zevallos, 1991), que constituiem fontes de Po e proporcionam aumento de $\mathrm{P}$ disponível mediante a exsudação de ácidos orgânicos em mecanismo similares aos desses mesmos ácidos oriundos da serapilheira (Wong et al., 2004), conforme descrição supracitada.

Assim, nos agrossistemas de cacau, a serapilheira acumulada e as raízes podem proporcionar acúmulos substantivos de Po na camada de 0-5 cm. Nesse caso, a quantidade média de Po lábil dos solos foi de $7,6 \mathrm{~kg} \mathrm{ha}^{-1}$. O conteúdo de $\mathrm{P}$ nas sementes é de aproximadamente $5 \mathrm{~kg} \mathrm{ha}^{-1}$ para uma produção de $1.000 \mathrm{~kg} \mathrm{ha}^{-1}$ (Gama-Rodrigues, 2004). Desse modo,

Quadro 5. Teores das frações de $\mathrm{P}$ inorgânico (Pi), orgânico (Po) e inorgânico + orgânico nas formas lábil e total do solo, nas camadas de 0-5 e 0-10 cm, sob agrossistemas de cacau

\begin{tabular}{|c|c|c|c|c|c|c|}
\hline \multirow{2}{*}{ Camada } & \multicolumn{2}{|c|}{$\mathrm{Pi}$} & \multicolumn{2}{|c|}{ Po } & \multicolumn{2}{|c|}{$\mathbf{P i}+\mathbf{P o}$} \\
\hline & Total & Lábil & Total & Lábil & Total & Lábil \\
\hline \multirow[t]{2}{*}{$\mathrm{cm}$} & & & \multicolumn{2}{|c|}{$\mathrm{mg} \mathrm{kg}^{-1}$} & & - \\
\hline & & & \multicolumn{2}{|c|}{ Floresta natural } & & \\
\hline $0-5$ & $212,8 \mathrm{a}$ & $3,8 \mathrm{a}$ & $44,8 \mathrm{a}$ & $17,1 \mathrm{a}$ & 257,6 a & 20,9 a \\
\hline $0-10$ & $193,4 \mathrm{~b}$ & $2,5 \mathrm{~b}$ & $29,6 \mathrm{~b}$ & $9,9 \mathrm{~b}$ & $223,0 \mathrm{~b}$ & $12,4 \mathrm{~b}$ \\
\hline \multicolumn{7}{|c|}{ Cacau renovado eritrina } \\
\hline $0-5$ & $338,2 \mathrm{a}$ & $6,1 \mathrm{a}$ & 60,4 a & $15,1 \mathrm{a}$ & 398,6 a & 21,1 a \\
\hline $0-10$ & $227,7 \mathrm{~b}$ & $5,5 \mathrm{~b}$ & $39,6 \mathrm{~b}$ & $7,0 \mathrm{~b}$ & $267,3 \mathrm{~b}$ & $12,5 \mathrm{~b}$ \\
\hline \multicolumn{7}{|c|}{ Cacau renovado cabruca } \\
\hline $0-5$ & $262,0 \mathrm{a}$ & $4,2 \mathrm{a}$ & $22,3 \mathrm{a}$ & $12,8 \mathrm{a}$ & $284,2 \mathrm{a}$ & $17,0 \mathrm{a}$ \\
\hline $0-10$ & $206,6 \mathrm{~b}$ & $3,5 \mathrm{~b}$ & $16,7 \mathrm{~b}$ & $3,5 \mathrm{~b}$ & $223,9 \mathrm{~b}$ & $7,0 \mathrm{~b}$ \\
\hline \multicolumn{7}{|c|}{ Cacau antigo cabruca } \\
\hline $0-5$ & 314,2 a & $5,8 \mathrm{a}$ & 179,3 a & $10,8 \mathrm{a}$ & 493,5 a & 16,7 a \\
\hline $0-10$ & $274,3 \mathrm{~b}$ & $4,6 \mathrm{~b}$ & $121,2 \mathrm{~b}$ & $7,9 \mathrm{~b}$ & $395,4 \mathrm{~b}$ & $12,4 \mathrm{~b}$ \\
\hline \multicolumn{7}{|c|}{ Cacau antigo eritrina } \\
\hline $0-5$ & $1.207,0 \mathrm{a}$ & 15,0 a & 461,8 a & $16,3 \mathrm{a}$ & $1.668,8 \mathrm{a}$ & 31,3 a \\
\hline $0-10$ & $705,7 \mathrm{~b}$ & $13,1 \mathrm{~b}$ & $390,4 \mathrm{~b}$ & $10,6 \mathrm{~b}$ & $1.096,0 \mathrm{~b}$ & $23,7 \mathrm{~b}$ \\
\hline \multicolumn{7}{|c|}{ Cacau jardim clonal } \\
\hline $0-5$ & 881,9 a & 38,6 a & 391,0 a & $19,1 \mathrm{a}$ & $1.273,0 \mathrm{a}$ & 57,7 a \\
\hline 010 & $774,1 \mathrm{~b}$ & $30,3 \mathrm{~b}$ & $212,2 \mathrm{~b}$ & $10,9 \mathrm{~b}$ & $986,3 \mathrm{~b}$ & $41,1 \mathrm{~b}$ \\
\hline
\end{tabular}

As médias seguidas de letras iguais, minúsculas na coluna, não diferem entre si pelo teste $\mathrm{F}$ a $5 \%$. 
a quantidade de Po lábil corresponde a 1,52 vez aquela que é exportada nas sementes. Esses resultados evidenciam que formas orgânicas de $\mathrm{P}$ potencialmente mineralizáveis podem constituir importantes fontes de suprimento do elemento para as plantas de cacau. Assim, estudos futuros sobre Po podem auxiliar no aperfeiçoamento do sistema de recomendação de fertilizantes fosfatados para a cultura do cacaueiro, baseado apenas na análise de $\mathrm{P}$ disponível (Mehlich1) na camada de 0-20 cm (Cabala-Rosand et al., 1988).

\section{CONCLUSÕES}

1. Nos agrossistemas de cacau, os Latossolos apresentaram menor teor de Po total, Po lábil e P microbiano do que os Cambissolos.

2. Nos Latossolos, os solos sob agrossistemas de cacau apresentaram maior teor de Po total, $\mathrm{P}$ microbiano e $\mathrm{P}$ disponível, mas menor teor de Po lábil do que o solo sob floresta natural.

3. O P disponível correlacionou-se positivamente com o Po (total, lábil e microbiano), e o Po total, com o P microbiano.

4. O Po lábil predominou amplamente sobre a fração inorgânica lábil, especialmente nos Latossolos.

\section{LITERATURA CITADA}

ALVARENGA, M.I.N. \& DAVIDE, A.C. Características físicas e químicas de um Latossolo Vermelho-Escuro e a sustentabilidade de agroecossistemas. R. Bras. Ci. Solo, 23:933-942, 1999

ALVAREZ V., V.H.; NOVAIS, R.F.; BARROS, N.F.; CANTARUTTI, R.B. \& LOPES, A.S. Interpretação dos resultados das análises de solos. In: RIBEIRO, A.C.; GUIMARÃES, P.T.G. \& ALVAREZ V., V.H., eds Recomendações para o uso de corretivos e fertilizantes em Minas Gerais. $5^{\mathrm{a}}$ Aproximação. Viçosa, MG, CFSEMG, 1999. 359p.

ANDERSON, J.N. \& INGRAM, J.S.I. Tropical soil biology and fertility: A handbook of methods. Wallingford, CAB International, 1996. 171p.

ACQUAYE, D.K. Some significance of soil organic phosphorus mineralization in the phosphorus nutrition of cocoa in Ghana. Plant Soil, 19:65-80, 1963.

BATAGLIA, O.C.; FURLANI, A.M.C.; TEIXEIRA, J.P.F.; FURLANI, P.R. \& GALLO, J.R. Métodos de análise química de plantas. Campinas, Instituto Agronômico, 1983. 48p. (Boletim técnico, 78)

BOWMAN, R.A. A sequential extraction procedure with concetrated sulfuric acid and diluted base for soil organic phosphorus. Soil Sci. Soc. Am. J., 53:326-366, 1989.
BOWMAN, R.A. \& COLE, C.V. Transformation of organic phophorus substrates in soil as evaluated by $\mathrm{NaHCO}_{3}$ extraction. Soil Sci., 125:95-101, 1978.

BROOKS, P.C.; POWLSON, D.S. \& JENKISON, D.S Phosphorus in the soil microbial biomass. Soil Biol. Biochem., 16:169-175, 1984.

CABALA-ROSAND, P.; SANTANA, M.B.M.; SANTANA, C.J.L.; CHEPOTE, R.E. \& NAKAYAMA, L.H. Utilização de adubos e corretivos na cultura do cacau. In: MANUAL do extensionista. Ilhéus, CEPLAC/CEPEC/, 1988. p.1-22.

CADE-MENUN, B.J. Characterizing phosphorus in environmental and agricultural samples by ${ }^{31} \mathrm{P}$ nuclear magnetic resonance spectroscopy. Talanta, 66:359-371, 2005 .

COMERFORD, N.B.; CROPPER, W.; GRIERSON, P.F.; ARAUJO, Q. \& JOSE, S. Modeling P bioavailability and uptake in agroforestry systems. In: GAMA-RODRIGUES, A.C.; BARROS, N.F.; GAMA-RODRIGUES, E.F.; FREITAS, M.S.M.; VIANA, A.P.; JASMIN, J.M.; MARCIANO, C.R. \& CARNEIRO, J.G.A., eds. Sistemas agroflorestais: Bases científicas para o desenvolvimento sustentável. Campos dos Goytacazes, Universidade Estadual do Norte Fluminense, 2006. p.303-315.

CONDRON, L.M.; MOIR, J.O.; TIESSEN, H. \& STEWART, J.W.B. Critical evaluation of methods for determining total organic phosphorus in tropical soils. Soil Sci. Soc. Am. J., 54:1261-1266, 1990 .

CUNHA, G.M.; GAMA-RODRIGUES, A.C.; COSTA, G.S. \& VELLOSO, A.C.X. Fósforo orgânico em solos sob florestas montanas, pastagens e eucalipto no norte fluminense. R. Bras. Ci. Solo, 31:667-671, 2007.

DUDA, G.P. Conteúdo de fósforo microbiano, orgânico e biodisponível em diferentes classes de solos. Itaguaí, Universidade Federal Rural do Rio de Janeiro, 2000. 158p. (Tese de Doutorado)

DUDA, G.P.; GUERRA, J.G.M.; MONTEIRO, M.T.; DE-POLLI H. \& TEIXEIRA, M.G. Perennial herbaceous legumes as live soil mulches and their effects on $\mathrm{C}, \mathrm{N}$ and $\mathrm{P}$ of the microbial biomass. Sci. Agric., 60:139-147, 2003.

EMPRESA BRASILEIRA DE PESQUISA AGROPECUÁRIA EMBRAPA. Centro Nacional de Pesquisa de Solos. Manual de métodos de análise de solo. 2.ed. Rio de Janeiro, 1997. 212p.

FASSBENDER, H.W.; ALPÍZAR, L.; HEUVELDOP, J.; FOSTER, H. \& ENRÍQUEZ, G. Modelling agroforestry systems of cacao (Theobroma cacao) with laurel (Cordia alliodora) and poro (Erythrina poeppigiana) in Costa Rica. III. Cycles of organic matter and nutrients. Agrofor. Syst., 6:49-62, 1988

FONTES, A.G. Ciclagem de nutrientes em sistemas agroflorestais de cacau no sul da Bahia. Campos dos Goytacazes, Universidade Estadual do Norte Fluminense, 2006. 71p. (Tese de Doutorado)

GAMA-RODRIGUES, A.C. \& CADIMA-ZEVALLOS, A. Efectos de fertilización sobre sistema radicular de cacao en suelos de "tabuleiros" del sur de Bahia, Brasil. Turrialba, 41:135141, 1991. 
GAMA-RODRIGUES, A.C. \& MIRANDA, R.C.C. O papel da chuva no fornecimento e reciclagem de nutrientes em um agrossistema de cacau do sul da Bahia, Brasil. Turrialba, 41:598-606, 1991a.

GAMA-RODRIGUES, A.C. \& MIRANDA, R.C.C. Efeito da chuva na liberação de nutrientes do folhedo num agrossistema de cacau do sul da Bahia. Pesq. Agropec. Bras., 26:1345-1350, 1991b.

GAMA-RODRIGUES, A.C. Ciclagem de nutrientes em sistemas agroflorestais na região tropical: funcionalidade e sustentabilidade. In: MÜLLER, M.W.; GAMARODRIGUES, A.C.; BRANDÃO, I.C.S.F.L. \& SERÓDIO, M.H.C.F., eds. Sistemas agroflorestais, tendência da agricultura ecológica nos trópicos: Sustento da vida e sustento de vida. Ilhéus, SBSAF/CEPLAC/UENF, 2004. p.64-84.

GEORGE, T.S.; GREGORY, P.J.; ROBINSON, J.R.; BURESH, R.J. \& JAMA, B. Utilization of soil organic P by agroforestry and crop species in the field, Western Kenya. Plant Soil, 246:53-63, 2002.

GOMES, J.B.V.; CURI, N.; MOTTA, P.E.F.; KER, J.C.; MARQUES, J.J.G.S.M. \& SCHULZE, D.G. Análise de componentes principais de atributos físicos, químicos e mineralógicos de solos do bioma Cerrado. R. Bras. Ci. Solo, 28:137-154, 2004.

GRESSEL, N. \& McCOLL, J.G. Phosphorus mineralization and organic matter decomposition: A critical review. In: CADISCH, G. \& GILLER, K.E., eds. Driven by nature: Plant litter quality and decomposition. Wallingford, CAB International, 1997. p.297-309.

GRIERSON, P.F.; SMITHSON, P.; NZIGUHEBA, G.; RADERSMA, S. \& COMERFORD, N.B. Phosphorus dynamics and mobilization by plants. In: van NOORDWISK, M.; CADISCH, G. \& ONG, C.K., eds. Below-ground interactions in tropical agroecosystems: Concepts and models with multiple plant components. Wallingford, CAB International, 2004. p.127-142.

GUERRA, J.G.M.; FONSECA, M.C.C.; ALMEIDA, D.J.; DEPOLLI, H. \& FERNANDES, M.S. Conteúdo de fósforo da biomassa microbiana de um solo cultivado com Brachiaria decumbens Staff. Pesq. Agropec. Bras., 30:543-551, 1995.

GUERRA, J.G.M.; ALMEIDA, D.J.; SANTOS, G.A. \& FERNANDES, M.S. Conteúdo de fósforo orgânico em amostras de solos. Pesq. Agropec. Bras., 31:291-299, 1996.

KUMMEROW, J.; KUMMEROW, M. \& SILVA, W.S. Fine root growth dynamics in cacao (Theobroma cacao). Plant Soil, 65:193-201, 1982.

LEHMANN, J.; GÜNTHER, D.; MOTA, M.S.; ALMEIDA, M.P.; ZECH, W. \& KAISER, K. Inorganic and organic soil phosphorus and sulfur pools in an Amazonian multistrata agroforestry system. Agrofor. Syst., 53:113-124, 2001.

MATOS, E.S.; MENDONÇA, E.S.; VILLANI, E.M.A.; LEITE, L.F.C. \& GALVÃO, J.C.C. Formas de fósforo no solo em sistemas de milho exclusivo e consorciado com feijão sob adubação orgânica e mineral. R. Bras. Ci. Solo, 30:625632, 2006.
MÜLLER, M.W. \& GAMA-RODRIGUES, A.C. Sistemas agroflorestais com cacaueiro. In: VALLE, R.R., ed. Ciência, tecnologia e manejo do cacaueiro. Ilhéus, CEPLAC, 2007. p.246-271.

MURPHY, J. \& RILEY, J.P. A modified single solution method for the determination of phosphate in natural waters. Anal. Chem. Acta, 27:31-36, 1962.

NOVAIS, R.F.; SMYTH, T.J. \& NUNES, F.N. Fósforo. In: NOVAIS, R.F.; ALVAREZ V., V.H.; FERNANDES, N.; FONTES, R.L.; CANTARUTTI, R.B. \& NEVES, J.C.L., eds. Fertilidade do solo. Viçosa, MG, Sociedade Brasileira de Ciência do Solo, 2007. p.471-550.

SANTANA, M.B.M.; CABALA-ROSAND, P. \& SANTANA, C.J.L. Exigências nutricionais e uso de fertilizantes em sistemas de produção de cacau. Ilhéus, CEPEC/CEPLAC, 1988.

SANTANA, M.B.M.; CABALA-ROSAND, P. \& SERÓDIO, M.H. Reciclagem de nutrientes em agrossistemas de cacau. Agrotrópica, 2:68-74, 1990.

SILVA, I.R. \& MENDONÇA, E.S. Matéria orgânica do solo. In: NOVAIS, R.F.; ALVAREZ V., V.H.; FERNANDES, N.; FONTES, R.L.; CANTARUTTI, R.B. \& NEVES, J.C.L., eds. Fertilidade do solo. Viçosa, MG, Sociedade Brasileira de Ciência do Solo, 2007. p.275-374.

SZOTT, L.T. \& MELENDEZ, G. Phosphorus availability under annual cropping, alley cropping, and multistrata agroforestry systems. Agrofor. Syst., 53:125-1132, 2001.

TER BRAAK, C.J.F. Canonical correspondence analysis: A new eigenvector technique for multivariate direct gradient analysis. Ecology, 67:1167-1179, 1986.

VANCE, E.D.; BROOKES, P.C. \& JENKINSON, D.S. An extraction method for measuring soil microbial biomass C. Soil Biol.Biochem., 19:703-707, 1987.

VELOSO, H.P.; RANGEL FILHO, A.L.R. \& LIMA, J.C.A. Classificação da vegetação brasileira adaptada a um sistema universal. Rio de Janeiro, IBGE, 1991. 124p.

WICK, B.; TIESSEN, H. \& MENEZES, R. Land use changes following the conversion of the natural vegetation into silvo-pastoral systems in semi-arid NE Brazil. Plant Soil, 222:59-70, 1998.

WONG, M.T.F.; HAIRIAH, K. \& ALEGRE, J. Managing soil acidity and aluminium toxicity in tree-based agroecosystems. In: van NOORDWISK, M.; CADISCH, G. \& ONG, C.K., eds. Below-ground interactions in tropical agroecosystems: Concepts and models with multiple plant components. Wallingford, $\mathrm{CAB}$ International, 2004. p.143-156.

ZAIA, F.C. Frações de fósforo do solo sob diferentes coberturas vegetais no norte fluminense e em plantios de cacau no sul da Bahia. Campos dos Goytacazes, Universidade Estadual do Norte Fluminense, 2005. 89p. (Tese de Mestrado) 